\title{
Time-Based Procurement
}

ZHAOLIN LI

The University of Sydney Business School, NSW 2006, Australia

erick.li@sydney.edu.au

\section{LUSHENG SHAO}

Faculty of Business and Economics, The University of Melbourne, VIC 3010, Australia

lusheng.shao@unimelb.edu.au

February 7, 2015 


\title{
Time-Based Procurement
}

\begin{abstract}
This study investigates the design of a time-based procurement contract when a supplier possesses private information about intrinsic completion time and may choose to exert time reduction effort. We first derive the optimal (complex) contract for the buyer, and then evaluate the performance of a (simple) fixed-price and fixed-time (FPFT) contract. Our analysis shows that the optimal time-based contract and the performance of an FPFT contract depend largely on time reduction forms. Specifically, if time reduction follows a multiplicative model, the optimal contract induces the intrinsically slow supplier to reduce time, and offers a fixed payment to the intrinsically fast supplier. This result is opposite to that which occurs when time reduction follows an additive model. We also show that, in a multiplicative model, the FPFT contract achieves at least eight-ninth of the available surplus for the buyer, whereas the performance of an FPFT contract deteriorates dramatically in an additive model. In response to this underperformance, we then propose an enhanced linear contract, and demonstrate that, using this contract, the buyer loses no more than one-sixteenth of the available surplus. Our results shed light on the important effect of time reduction forms on time-based contract design.

[Keywords: Delivery time, procurement contract design, incomplete information, hidden action]
\end{abstract}

\section{INTRODUCTION}

Time is money. Intense global competition and rapid technological advances have made speed a competitive advantage. To succeed, companies must identify new opportunities and launch new products or services to the marketplace ahead of their competitors. Perhaps in no industry does speed matter as much as in the consumer electronics industry. For example, a delay in launching a product can result in a decline in sales ranging from $5.92 \%$ to $10.99 \%$ (Hendricks \& Singhal, 2008) and a loss of $5.25 \%$ of the firm's share price (Hendricks \& Singhal, 1997). The construction industry is another good example in which time and speed matter. Since a typical construction project requires debt financing, any reduction in time could directly lower the borrowing cost. According to Konchar and Sanvido (1998), the median construction cost in 
the USA is $\$ 1,140$ per square meter, and the median construction speed is 438 square meters per month. Consider a residential project with a size of 100,000 square meters. Suppose that $75 \%$ of the construction cost is debt financed, and the prevalent interest rate of commercial loans is $4.8 \%$ per annum. Then a property developer such as D. R. Horton could save as much as $\$ 342,000$ in interest expenses if the completion time is reduced by just one month.

In the face of increasing time-to-market pressure, numerous organizations are turning to outsourcing to gain competitive advantage over their competitors. For example, Pannos Kalaritis, the vice president of operations for Irix Pharmaceuticals, says that outsourcing process development can accelerate a drug's development by allowing a pharmaceutical company to continue research, while a contractor works on process optimization (Lerner 2002). Time has been an important criterion when organizations source services for critical construction projects, new product design, enterprise resource planning (ERP) software, and so forth. In public sectors, the time to complete a project significantly affects consumers' wellbeing and social welfare. For example, the social cost caused by a delay in highway repair jobs could be as high as several million dollars per day (Lewis \& Bajari, 2011).

A contractor (or supplier) can usually speed up job completion by shifting resources from other jobs, hiring more workers, using equipments more intensively, or scheduling overtime. After recognizing such possibilities, a number of organizations offer explicit time incentive to their contractors. This paper studies a situation in which a buyer purchases an item (product or service) from a supplier, and benefits from fast delivery. The question is how to design time-based incentive that is offered to the supplier. This same model also applies to an outsourcing setting, in which a firm outsources a job (project or process) to a contractor and is concerned with designing a time-based contract to incentivize the contractor to complete the job as early as possible.

Incentive-based contracts have seen widespread applications in practice, and can make time reduction rewarding for both buyers and suppliers. For example, on January 17, 1994, a 6.8-magnitude earthquake struck the Los Angeles basin near the suburb of Northbridge and caused 60 deaths, thousands of injuries, and billions of dollars in property damage. Nowhere was the destructive power of nature more evident than in the collapsed sections of the freeway system that disrupted the daily commute of an estimated 1 million local residents. The Northbridge earthquake posed one of the greatest challenges to California Department 
of Transportation (CalTrans) in its nearly 100-year history. To expedite the recovery process, Governor Pete Wilson signed an emergency declaration allowing CalTrans to streamline contracting procedures and offer attractive incentive for completing work ahead of schedule. The time incentive scheme proved to be a powerful motivator for the freeway reconstruction contractors. C. C. Myers, Inc., pulled out a stunning feat by repairing the damaged freeways 74 days ahead of schedule and was awarded a $\$ 14.8$ million bonus (Baxter 1994). Despite the difficulties and expenses incurred by around-the-clock freeway building, most local residents cheered CalTran's quake recovery efforts.

In this study, we consider a procurement model in which a supplier possesses private information about intrinsic completion time and is able to invest in time reduction effort. In practice, it is difficult (and sometimes impossible) for a buyer to observe the effort exerted by the supplier. However, the actual time when the supplier delivers the good or service is always observable and verifiable. Therefore, the buyer can write a time-based contract to achieve the optimal procurement outcome. Drawing on the principal-agent literature, this paper is concerned with how to incentivize the supplier to reduce time in such a situation where the supplier has private initial completion time and private time reduction effort.

An important question is how to model time reduction since this will directly affect the buyer's utility, and hence the optimal time-based procurement contract. We consider two time reduction models: (1) multiplicative model, in which the reduced time is increasing in the intrinsic time with the same level of effort, implying that it is easier to reduce the completion time if the initial time is shorter; and (2) additive model, in which the reduced time does not depend on the intrinsic time. These two models are likely to emerge in various circumstances. Consider a multi-level residential building as an example. The use of fast-drying cements can reduce the drying time for each level by 1 day, which is independent of the intrinsic time. On the other hand, the use of concurrent design (e.g., Jayaram and Malhotra, 2010) or laddering technique (e.g., Larson and Gray, 2011) can also reduce the completion time. Laddering means that project activities are broken into segments so that the following activity can begin sooner and not delay the work. Suppose that it takes the contractor to trench, lay pipe, and refill in 9 days (with 3 days for each activity). The contractor can break these three activities into three segments such that it can start to lay pipe when the first segment of trenching is completed, start to refill when the first segment of laying pipe is completed, and so forth. The total time would reduce from 9 to 5 days. In this case, the reduced time is increasing in the intrinsic time. 
In summary, different options to reduce completion time can lead to the additive or multiplicative model.

For each model, we first design the optimal time-based procurement contract, and then assess the performance of a simple but often used contract, namely the fixed-price and fixed-time (FPFT) contract. Under this contract, the buyer agrees to pay the supplier a fixed payment if the supplier is able to complete the job within a fixed amount of time. The results and insights in this paper are rich. First, we demonstrate that whether the time reduction is multiplicative or additive significantly changes the form of optimal payment (as a function of actual completion time). In particular, when the time reduction follows an additive model, it is optimal for the buyer to induce a fast supplier to reduce time by using a convex payment function. In contrast, when the time reduction follows a multiplicative model, it is optimal for the buyer to induce a slow supplier to reduce time by using a concave payment function. In practice, a convex payment function is reminiscent of a contract that offers a large bonus for each unit of reduced time; whereas a concave payment function is reminiscent of a contract that stipulates a firm delivery time. Both contract forms are being used in modern procurement systems (see Larson \& Gray, 2011; and Lewis \& Bajari, 2011). In this sense, our research provides a holistic economic explanation on the use of time-based incentive. In addition, the results suggest that the buyer may suffer long delays or high costs if it uses a payment function that is based on an incorrect assumption.

Second, we show that the performance of some simple contracts critically depends on the cost of exerting effort and whether time reduction is multiplicative or additive. In particular, when time reduction follows a multiplicative model, we demonstrate that by using an FPFT contract, the buyer may lose no more than $1 / 9$ of the achievable surplus secured by an optimal contract. However, when time reduction follows an additive model, the performance of an FPFT contract deteriorates especially when the cost of exerting effort is medium or high. In response to this underperformance, we propose an enhanced linear contract, and demonstrate that the performance ratio of this enhanced contract is at least 15/16.

Our results highlight the importance of identifying time reduction forms. In practice, it may be difficult for a buyer to recognize which model the time reduction follows. A rule of thumb is to consider whether the time that can be possibly reduced depends on the initial time. If the reduced time is affected by the intrinsic time, then time reduction is likely to follow a multiplicative model; otherwise, it may follow an additive model. Then based on the conjecture of time reduction, the buyer designs an appropriate time- 
based contract.

\section{Literature Review}

This study is broadly related to the literature on principal-agent models with incomplete information (which leads to adverse selection) and hidden action (which leads to moral hazard). For a general review on this topic, refer to Bolton and Dewatripont (2005). In the following, we will focus our attention on the most relevant articles, which study how to incentivize suppliers to reduce their production costs. The two influential papers in this area were written by Baron and Myerson (1982), where the agent's production cost is unobservable, and Laffont and Tirole (1986), where the agent's production cost is observable. In Laffont and Tirole (1986) and numerous subsequent studies, the agent's cost is assumed to be additive with respect to the agent's effort. Specifically, the agent privately observes the intrinsic cost and chooses to invest in cost reduction. The principal observes the agent's realized cost, which equals the intrinsic cost minus the agent's private effort.

Our study differs from the above literature in that we consider a procurement mechanism design problem with time reduction. Time reduction is distinct from cost reduction in that the former may be of multiplicative or additive form, while the latter is of additive form (as the literature suggests). More importantly, we find that the optimal payment function has contrasting properties for these two models, which has not been reported in the existing studies. Arguably, this paper is the first to study time-based contract design with incomplete information and hidden action.

In the operations management literature, several papers study the issue of on-time delivery, whereas we focus on inducing the supplier to reduce time. Grout and Christy (1993) study a just-in-time environment, in which the supplier determines when to start the production, and show that a bonus contract improves on-time delivery. Grout (1998) shows that the use of delivery windows does not always improve on-time delivery. Cachon and Zhang (2006) consider a procurement problem in which the supplier possesses private information about capacity cost. Recognizing that the reserved capacity determines the delivery time, Cachon and Zhang characterize the optimal procurement mechanisms and evaluate the performance of two simple contracts. Dai et al., (2012) study the contracting problem in a vaccine supply chain by integrating various industrial features such as product design, uncertain delivery, and time-sensitive demand. We contribute to 
this literature by identifying the impact of time reduction on optimal contracts and developing theoretical bounds on the forgone surplus when an FPFT contract is used.

The remainder of this paper is organized as follows: Section 2 presents the model setup, and Section 3 derives the optimal time-based contracts for the multiplicative model and the additive model. Then we evaluate the performance of the FPFT contract in Section 4, and finally we summarize the main findings and discuss the future research in Section 5. All the proofs are related to the Appendix. We also study a time-based auction to achieve the optimal procurement outcome if there are multiple suppliers competing for the buyer's business. This together with other extensions is provided in the Online Supplement.

\section{THE PROCUREMENT MODEL}

Consider a situation in which a time-sensitive buyer ("she") purchases an item (product or service) from a qualified supplier ("he"). Before interacting with the buyer, the supplier learns his intrinsic completion time $x$, which is the realization of a random variable $X$ with (normalized) support over the interval $[0,1]^{1}$. The cumulative distribution function (CDF) of $X$ is $F(x)$, and the probability density function (PDF) is $f(x)$, where $x \in[0,1]$. The realized intrinsic time $x$ is private to the supplier, and the buyer only knows the distribution of $X$.

In our model, the supplier can reduce his completion time from $x$ to $t(x, y)$ by exerting effort at level $y$, where $t(x, y)$ is a continuous bivariate function satisfying $t(x, 0)=x$ for any $x \in[0,1]$. In other words, without committing any effort, the supplier's delivery time is his intrinsic completion time. Although the value of $y$ is unknown to the buyer, she can always observe when the supplier delivers the item. Therefore, she can write a contract based only on the observed completion time. Let $P(t)$ be the payment that she pays to the supplier under the condition that the observed time is $t$. Intuitively, the optimal payment function must be a (weakly) decreasing function of completion time. We make the following assumption on $t(x, y)$ and $C(y)$.

Assumption 1 We assume: a) $\frac{\partial t(x, y)}{\partial x} \geq 0, \frac{\partial t(x, y)}{\partial y} \leq 0$; and $\left.b\right) C^{\prime}(y)>0, C^{\prime \prime}(y) \geq 0$ for $y>0, C(y) \geq 0$ for any $y$.

\footnotetext{
${ }^{1}$ A seemingly more general model may assume that the support of $X$ is an interval $[\underline{x}, \bar{x}]$, where $\underline{x}$ is the shortest time when the supplier can deliver the item without committing effort. Because $\underline{x}$ is a constant, we can normalize the support for the intrinsic time $X$ to be the unit interval. Therefore, the use of $[0,1]$ does not lose any generality.
} 
Part a) in Assumption 1 implies that the actual completion time is non-decreasing in the intrinsic time with any given effort level, and is non-increasing in the effort level with any given intrinsic time. Part b) states that the supplier's disutility function $C(y)$ is increasing and convex. We also assume that $C(y) \geq 0$ for $y \leq 0$, which implies that the supplier incurs a nonnegative disutility if he deliberately delays completion. Because the optimal payment function is a (weakly) decreasing function of the observed completion time, the supplier will deliver the good as soon as it is ready.

The buyer's (gross) utility is assumed to be $r-t$, where $r>1$ is a positive constant. As such, the buyer always attains positive utility even when the supplier's actual completion time is the maximum ${ }^{2}$. In the Online Supplement, we extend the analysis to the case where the buyer's utility is a concave and decreasing function of $t$ (which captures the possibility that a longer delay is more costly for the buyer). We find that the major insights of our analysis based on linear utility function remain intact.

The buyer's (net) utility is $u_{b}=r-t(x, y)-P(t)$, and the supplier's utility is $u_{s}=P(t)-C(y)$, where $P(t)$ is the transfer payment from the buyer to the supplier. It is straightforward to show that $u_{b}+u_{s}=$ $r-t(x, y)-C(y)$, which gives the supply chain surplus conditional on the type of supplier (i.e., the intrinsic time $x$ ) and the effort $y$. At this point, it would be interesting to compare in detail our time-based model with the cost-based model. Suppose, in a classic cost-based model, the buyer's utility is $r-P$ and the supplier's utility is $P-c(x, y)-e(y)$, where $c(x, y)$ is the actual cost, $x$ is the intrinsic cost, $y$ is the cost reduction effort, and $e(y)$ is the disutility associated with $y$. We observe that the actual cost $c$ affects the supplier's utility; whereas the actual time $t$ (the counterpart of $c$ ) does not affect the supplier's utility. Such a difference drastically reduces the performance of a fixed price contract in our time-based model. Specifically, Rogerson (2003) shows that a fixed price contract attains at least $75 \%$ of the achievable surplus; while we find that a FPFT contract could fail to achieve any achievable surplus (i.e., the performance ratio is 0 ). In addition, from a practical point of view, verifying cost is more difficult than verifying time: to verify the cost, the buyer often needs to hire an auditor to investigate whether the supplier includes some non-allowable expenses or inflates some allowable expenses; whereas verifying the time when the supplier delivers the item does not present any challenge. These nuanced distinctions imply that the results in a cost-based model may not

\footnotetext{
${ }^{2}$ A slightly more general formulation may assume that the buyer's (gross) utility is $r-a t$, where $r, a$ are positive constants with $r>a$. By scaling the disutility function properly, we can recover all of the results developed in this paper. Therefore, assuming that $a=1$ is without loss of generality.
} 
apply in a time-based setting as we discuss earlier.

Let $y(x)$ be the $x$-type supplier's optimal effort given the payment function $P(t)$. If a type- $x$ supplier pretends to be a type- $\hat{x}$ supplier, he must choose the effort level $y(\hat{x} \mid x)$ such that the actual completion time is the same as that achieved by a type- $\hat{x}$ supplier, which implies that

$$
t(x, y(\hat{x} \mid x))=t(\hat{x}, y(\hat{x}))
$$

By pretending to be a type- $\hat{x}$ supplier, the type- $x$ supplier attains a surplus of

$$
u_{s}(\hat{x} \mid x)=P(t(\hat{x}, y(\hat{x})))-C(y(\hat{x} \mid x)) .
$$

We focus on the truthful revelation mechanism, in which it is optimal for the supplier to truthfully reveal his type. Using the revelation principle (e.g., Baron and Myerson, 1982), we formulate the buyer's contract design problem as follows:

$$
\max _{P(\cdot), y(\cdot)} \int_{0}^{1}[r-t(x, y)-P(t(x, y))] d F(x)
$$

subject to the incentive compatibility constraints (IC) and individual rationality constraints (IR),

$$
\begin{aligned}
& \text { IC: } u_{s}(x \mid x) \geq u_{s}(\hat{x} \mid x), \quad \forall \hat{x}, x \in[0,1], \\
& \text { IR: } u_{s}(x \mid x) \geq 0, \quad \forall x \in[0,1] .
\end{aligned}
$$

The IC constraints indicate that truthful revelation is optimal for the supplier, and the IR constraints state that each type of the supplier can obtain at least his reservation profit which is normalized to be zero.

\section{OPTIMAL TIME-BASED CONTRACT}

In this section, we start by analyzing a model with generic functions of $t(x, y)$ and $C(y)$, but later, for tractability, we will focus on specific models to gain more insights into the optimal procurement contract. We solve the buyer's contract design problem in three steps. Since the solution approach is rather lengthy and complex, we only provide a sketch proof here, and relegate the details to the Appendix (see the proof of Lemma 1). 
Let $U_{s}(x)=u_{s}(x \mid x)$ denote the surplus that a type- $x$ supplier attains in the truth-revealing equilibrium. First, we apply the envelope theorem to derive the $U_{s}(x)$ function. Second, we replace $P(\cdot)$ in equation (3) with $U_{s}(\cdot)+C(y(\cdot))$. This step allows us to write down the virtual surplus function as follows:

$$
V(x, y)=r-t(x, y)-C(y)+\frac{C^{\prime}(y) \cdot \frac{\partial t(x, y)}{\partial x}}{\frac{\partial t(x, y)}{\partial y}} \frac{F(x)}{f(x)} .
$$

We observe that the virtual surplus equals the supply chain profit minus the information rent that must be paid to the supplier. As such, we can reformulate the buyer's problem as follows,

$$
\max _{P(\cdot), y(\cdot)} \int_{0}^{1} V(x, y) d F(x),
$$

subject to: $t(x, y(x))$ is a monotonic function of $x$.

We now solve the above problem by first ignoring the constraint. If the integrand $V(x, y)$ is maximized against $y$ for any given $x$, then the buyer's expected utility is also maximized. For any given $x \in[0,1]$, define an effort function as

$$
y^{*}(x)=\arg \max _{y}\{V(x, y)\}
$$

Because the term $\frac{\partial t(x, y)}{\partial x} / \frac{\partial t(x, y)}{\partial y}$ in equation (6) has a negative sign, we can conclude that $y^{*}(x) \leq \bar{y}(x)$, where $\bar{y}(x)=\arg \max _{y}\{r-t(x, y)-C(y)\}$ denotes the supply chain optimal effort. Third, we examine whether $t\left(x, y^{*}(x)\right)$ is a monotonic function of $x$. If $t\left(x, y^{*}(x)\right)$ is non-decreasing in $x$, then $y^{*}(x)$ is optimal for the problem in (7). We summarize the buyer's optimal payment in Lemma 1.

Lemma 1 If $\left(x, y^{*}(x)\right)$ is non-decreasing in $x$, then the optimal effort committed by the type- $x$ supplier is $y^{*}(x)$, and the optimal payment function satisfies

$$
P^{*}\left(t\left(x, y^{*}(x)\right)\right)=C\left(y^{*}(x)\right)-\int_{x}^{1} C^{\prime}\left(y^{*}(\tilde{x})\right) \frac{\frac{\partial t\left(\tilde{x}, y^{*}(\tilde{x})\right)}{\partial \tilde{x}}}{\frac{\partial t\left(\tilde{x}, y^{*}(\tilde{x})\right)}{\partial y^{*}(\tilde{x})}} d \tilde{x} .
$$

Equation (9) indicates that the supplier receives a time-based payment that equals the cost of exerting time reduction effort plus the information rent. We observe that Lemma 1 builds on an important condition that $t\left(x, y^{*}(x)\right)$ is a non-decreasing function of $x$. If $t\left(x, y^{*}(x)\right)$ is decreasing in $x$, we need to apply the "ironing and bunching" procedure, which is computationally intensive, to solve the optimal solution. In the Appendix, we derive a sufficient condition such that $t\left(x, y^{*}(x)\right)$ is non-decreasing in $x$. The sufficient 
condition is complex, and consists of two parts. The first part is related to the distribution of intrinsic completion time, which is satisfied if $F(x)$ is log-concave. The second part, which is related to the supplier's disutility $C(y)$ and time reduction $t(x, y)$, ensures that the supplier's equilibrium utility decreases in his intrinsic time. Intuitively, an intrinsically slow supplier should not attain higher utility than an intrinsically fast supplier in equilibrium. Thus, it is reasonable to assume that the primitives in the model satisfy the sufficient condition given in the Appendix.

A primary objective of this study is to investigate how different $t(x, y)$ functions may affect the optimal payment function. In order to better understand how time reduction affects the optimal contract, we focus on two primary cases: the multiplicative model with $t(x, y)=x-x y$, and the additive model with $t(x, y)=$ $x-y$. These two models represent different business settings as discussed in Section 1. A noteworthy difference between these two models is whether the reduced time depends on the intrinsic time. In the multiplicative model, the reduced time increases in the intrinsic time; whereas in the additive model, the reduced time is not affected by the intrinsic time. Interestingly, such a difference leads to the contrasting properties of optimal payment functions.

It is worth nothing that the effort level $y$ has different interpretations in these two cases. In the multiplicative model, $100 y \%$ denotes the percentage of reduction in time that the supplier achieves through his effort. We impose a constraint, $y \leq 1$, in this case to ensure that the supplier does not reduce time below zero. In the additive model, $y$ denotes the amount of time the supplier reduces through his effort. We impose a constraint, $y \leq x$, in this case to ensure that the supplier does not reduce time below zero. We also assume that the intrinsic time is uniformly distributed for both cases, i.e., $F(x)=x$ and $f(x)=1$ for any $0 \leq x \leq 1$. The uniform distribution satisfies the first part of the sufficient condition stated in the Appendix and is consistent with numerous prior studies (e.g., Laffont and Tirole,1986; and Rogerson, 2003).

\subsection{The Multiplicative Model}

Consider a multiplicative model with $t(x, y)=x-x y$. We assume that the supplier incurs disutility

$C(y)=\frac{k y^{2}}{1-y}$ when he achieves a $100 y \%$ time reduction for $0 \leq y \leq 1$, where $k>0$ is a constant. The choice of such a $C(\cdot)$ is mainly for tractability purpose. This particular $C(\cdot)$ function is convex and increasing, approaches infinity when $y$ approaches 1 , and satisfies the second part of the sufficient condition stated in the Appendix. These properties guarantee that the optimal solution is an interior solution. Let 
$G_{m}$ denote the buyer's optimal expected utility in the multiplicative model. Proposition 1 characterizes the optimal time-based procurement contract in the multiplicative model.

Proposition 1 The optimal time-based procurement contract for the multiplicative model has the following properties.

a) It induces the type- $x$ supplier to choose an effort level of $y_{m}(x)=\max \left(0,1-\sqrt{\frac{2 k}{x}}\right)$.

b) The buyer's optimal expected utility satisfies

$$
G_{m}= \begin{cases}r-\frac{1}{2}, & k \geq \frac{1}{2} \\ r+2 k-\frac{2 k^{2}}{3}-\frac{4}{3} \sqrt{2 k}, & k \leq \frac{1}{2}\end{cases}
$$

c) If $k \geq \frac{1}{2}$, the optimal payment function is $P_{m}(t)=0$ for all $t \in[0,1]$, whereas if $k \leq \frac{1}{2}$, the optimal payment function satisfies

$$
P_{m}(t)= \begin{cases}\sqrt{2 k}+\sqrt{8 k^{3}}-4 k, & 0 \leq t \leq 2 k, \\ \sqrt{2 k}+\sqrt{8 k^{3}}-\frac{t}{2}-\frac{2 k^{2}}{t}-2 k, & 2 k \leq t \leq 1,\end{cases}
$$

which is a continuous, concave, and decreasing function of $t$.

Proposition 1 shows that, if $k \geq \frac{1}{2}$, implying that the cost of exerting private effort is too high (relative to the value of time reduction), it is then optimal for the buyer not to offer any time incentive. Thus, the supplier's private effort is zero, and the buyer's expected utility equals his willingness to pay subtracted from the expected time cost (i.e., $r-\frac{1}{2}$ ). In contrast, when $k<\frac{1}{2}$, the buyer's optimal contract induces the supplier with an intrinsic time longer than $2 k$ to reduce time. The buyer induces a greater time reduction from the supplier with a longer intrinsic time. The supplier with an intrinsic time shorter than $2 k$ does not exert any time-reduction effort. From a) in Proposition 1, we can show that the final completion time (after time reduction) is given by $t_{m}\left(x, y_{m}(x)\right)=\min (x, \sqrt{2 k x})$. In addition, all supplier types (except the slowest supplier) receive a positive information rent. The optimal time-payment function begins as a constant and decreases when the completion time exceeds the threshold $2 k$.

In Figure 1, we depict the optimal effort as a function of the supplier's intrinsic time and the optimal payment as a function of the actual completion time (where $k=0.25$ ). We observe from Sub-figure 1(a) that the optimal effort remains unchanged as $x$ increases from 0 to 0.5 , and then increases as $x$ increases from 0.5 to 1 . Sub-figure 1(b) illustrates that the optimal payment is the same for any $t \in[0,0.5]$ and decreases in $t$ where $t \in(0.5,1]$. 


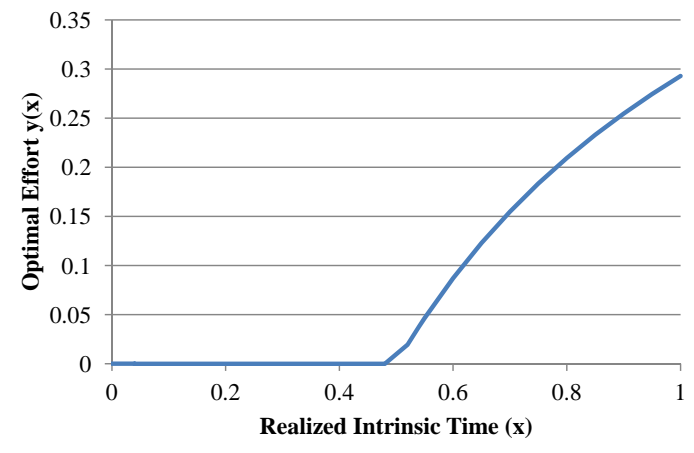

(a) Optimal Effort Induced By Buyer

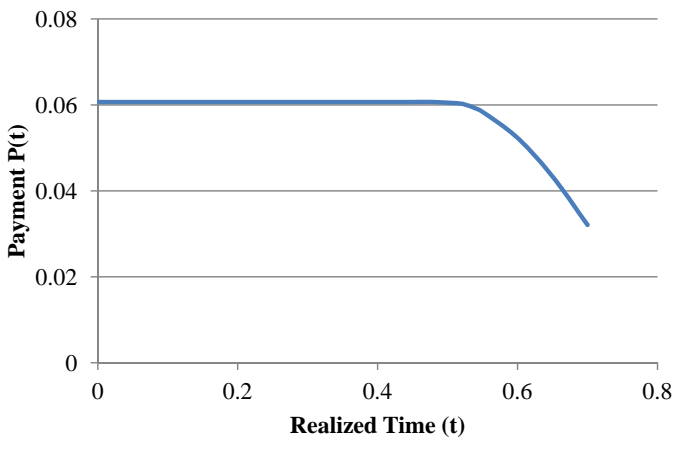

(b) Optimal Payment Function

Figure 1: Properties of optimal contract for the multiplicative model

\subsection{The Additive Model}

In the additive model, the time reduction is of the additive form with $t(x, y)=x-y$. Following Laffont and Tirole (1986) and Rogerson (2003), we assume that the supplier incurs disutility $C(y)=\frac{y^{2}}{4 h}$ when he reduces the completion time by $y$, where $h>0$ is a constant. One may wonder why different disutility functions are used in these two models. The reason is two-fold: first, since our interest is to compare the optimal payment structure between the multiplicative model and the additive model, we use different disutility functions mainly for technical convenience; second and more importantly, the disutility functions do not affect the qualitative results given in this paper. We have tried different forms of disutility function such as a cubic function and an exponential function, and found that the main structure of optimal payment function remains unchanged. However, using these functions leads to complicated and implicit expressions of the optimal effort and payment function, thus, we have to use numerical computations.

Let $G_{a}$ denote the buyer's optimal expected utility in the additive model. Proposition 2 summarizes the optimal contract in the additive model.

Proposition 2 The optimal time-based procurement contract for the additive model has the following properties.

a) It induces the type-x supplier to choose an effort level of

$$
y_{a}(x)= \begin{cases}x, & 0 \leq x \leq h, \\ 2 h-x, & h \leq x \leq 2 h, \\ 0, & x \geq 2 h .\end{cases}
$$


b) The buyer's optimal expected utility satisfies

$$
G_{a}= \begin{cases}r+\frac{h^{2}}{3}-\frac{1}{2}, & 0<h<\frac{1}{2} \\ r+h+\frac{1}{12 h}-\frac{h^{2}}{3}-1, & \frac{1}{2} \leq h<1 \\ r-\frac{1}{4 h}, & h \geq 1\end{cases}
$$

c) When $0<h<\frac{1}{2}$, the optimal payment function satisfies

$$
P_{a}(t)= \begin{cases}0, & t \geq 2 h \\ \frac{h}{2}-\frac{t}{2}+\frac{t^{2}}{8 h}, & t \leq 2 h .\end{cases}
$$

When $\frac{1}{2} \leq h<1$, the optimal payment function satisfies

$$
P_{a}(t)= \begin{cases}0, & t>1-h, \\ 1-\frac{1}{4 h}-\frac{h}{2}-\frac{t}{2}+\frac{t^{2}}{4 h}, & t \leq 1-h .\end{cases}
$$

When $h \geq 1$, the optimal payment function satisfies $P_{a}(0)=\frac{1}{4 h}$ and $P_{a}(t)=0$ for any $t>0$.

Proposition 2 shows that the optimal effort $y_{a}(x)$ is a piece-wise linear function of $x$, which increases first, then decreases, and finally remains unchanged (with zero). The optimal effort is largest when the supplier's type is $h$. This suggests that the buyer induces the fast supplier to reduce time, making the fast supplier even faster. From $y_{a}(x)$, we can calculate the final completion time (after time reduction) as follows:

$$
t_{a}\left(x, y_{a}(x)\right)=x-y_{a}(x)= \begin{cases}0, & 0 \leq x \leq h \\ 2 x-2 h, & h \leq x \leq 2 h \\ x, & x \geq 2 h\end{cases}
$$

It is optimal for the buyer to incentivize all the types no greater than $h$ to reduce the completion as much as possible. As the intrinsic time is long (i.e., when $x \in(h, 2 h]$ ), the supplier does not have much incentive to reduce the completion time. As a result, the final completion time is positive (due to $2 x-2 h>0$ ). In the extreme case where $x \geq 2 h$, the buyer does not provide any time reduction incentive to the supplier.

Using $h=0.2$, we depict the optimal effort and payment function for the additive model in Figure 2, which shows that the buyer induces the fast supplier to reduce time and offers a fixed (zero) payment to the slow supplier. The optimal payment function is continuous, convex, and decreasing in realized time.

Propositions 1 and 2 reveal some contrasting properties of the optimal payment function. The reasons that drive these contrasting results are noteworthy. In the multiplicative model, the slow supplier (with large $x$ ) achieves a larger time reduction than the fast supplier does when both exert the same level of effort. In other words, inducing the slow supplier to reduce time is less costly than inducing the fast supplier to do the 


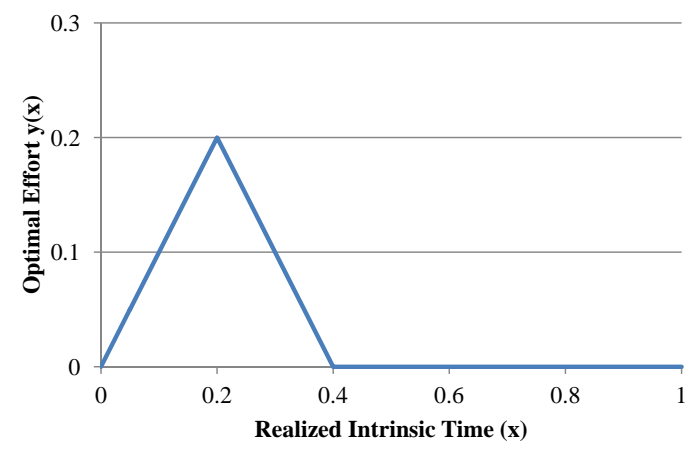

(a) Optimal Effort Induced By Buyer

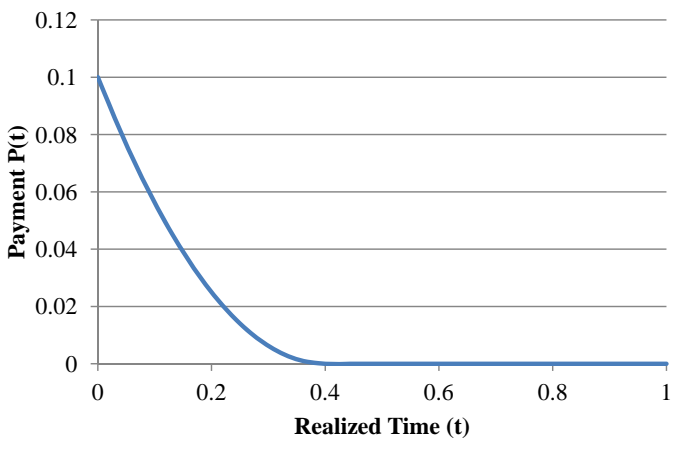

(b) Optimal Payment Function

Figure 2: Properties of optimal contract for the additive model

same. With a concave payment function, the fast supplier receives an information rent but does not take any action to reduce time. In the additive model, the same level of private effort achieves the same amount of time reduction for any supplier type. If the buyer offers time incentive, the fast supplier is more capable of meeting the time requirement. Therefore, it is optimal for the buyer to provide a large information rent to induce the fast supplier to reduce time and to provide no incentive to the slow supplier.

These contrasting results suggest that the buyer may suffer long delays or high costs if she uses a payment function that is based on an incorrect assumption. For example, if the buyer uses the convex decreasing payment function when time reduction is multiplicative, she suffers either long delay because the convex payment function fails to motivate the slow supplier to reduce time, or high cost because the payment for fast delivery is too generous. Similar problems occur if the buyer uses the concave decreasing payment function when time reduction is additive. The buyer suffers either long delays because of the failure to induce the fast supplier to exert time-reduction effort or high cost because of the generous deadline that allows the slow supplier to receive rent.

\section{THE FPFT CONTRACT}

The optimal payment functions derived in Propositions 1 and 2 are complex and non-linear. However, the real world incentive schemes appear to take less extreme forms than do the finely tuned rules predicted by economic theory (Holmstrom and Milgrom 1987, page 304). The simple fixed-price and fixed-time (FPFT) contract has frequently been used in modern project management (see Chapter 12 of Larson and Gray, 2011). 
Under such a contract, the supplier receives a fixed payment $P_{0}$ upon successfully delivering the good before time $t_{0}$. To determine how well this FPFT contract performs relative to the optimal contract, we first derive the optimal FPFT contract $\left(P_{0}, t_{0}\right)$.

Similar to the derivation of optimal time-based contract in Section 3, we begin with the generic model without assuming specific function forms on time reduction and the supplier's disutility. Then, we study the FPFT contract for both the multiplicative and additive model. Lemma 2 characterizes the optimal FPFT contract in general cases.

Lemma 2 The optimal FPFT contract can be found by solving the following three equations.

$$
\frac{d C\left(y\left(t_{0}\right)\right)}{d t_{0}}=1-F\left(t_{0}\right), \quad t\left(1, y\left(t_{0}\right)\right)=t_{0}, \quad P_{0}=C\left(y\left(t_{0}\right)\right) .
$$

Under the optimal FPFT contract, the supplier with an intrinsic time shorter than the maximum allowable time does not commit any time reduction effort, but the supplier with an intrinsic time longer than the maximum allowable time takes action to reduce time such that the realized completion time exactly equals the maximum allowable time. As such, there is a positive probability (i.e., $\operatorname{Pr}\left(x>t_{0}\right)=1-F\left(t_{0}\right)$ ) that the supplier delivers the product or service exactly when the deadline expires. The optimal fixed payment makes the slowest supplier type indifferent between committing and not committing effort.

\subsection{The Multiplicative Model}

Now consider the multiplicative model with $t(x, y)=x-x y$. Using Lemma 2, we derive the optimal FPFT contract in this case as follows.

Proposition 3 The optimal FPFT contract for the multiplicative model has the following properties.

a) The buyer's optimal fixed time is

$$
t_{0}^{m}= \begin{cases}\frac{k+\sqrt{4 k+k^{2}}}{2}, & k \leq \frac{1}{2}, \\ 1, & k \geq \frac{1}{2},\end{cases}
$$

and the optimal fixed payment is $P_{0}^{m}=C\left(1-t_{0}^{m}\right)=\frac{k\left(1-t_{0}^{m}\right)^{2}}{t_{0}^{m}}$.

b) The buyer's optimal expected utility that can be achieved by implementing an FPFT contract is given by

$$
G_{l}^{m}= \begin{cases}r+\frac{5}{2} k-\frac{k^{2}}{4}-\frac{k+4}{4} \sqrt{4 k+k^{2}}, & k \leq \frac{1}{2} \\ r-\frac{1}{2}, & k \geq \frac{1}{2}\end{cases}
$$


Proposition 3 shows that if the effort cost is high (when $k \geq 1 / 2$ ), then it is optimal for the buyer to simply set the maximum allowable time to be 1 without paying anything to the supplier. In this case, the supplier does not exert any effort to reduce the completion time. On the other hand, if the effort cost is low (when $k<1 / 2$ ), then the buyer motivates the supplier to exert some effort to reduce the completion time. The maximum allowable time increases as $k$ increases, suggesting that it is sensible to set a long deadline if the cost of reducing time is high.

To benchmark the performance of the FPFT contract, we introduce the concept of "achievable surplus". Note that without any time incentive, the buyer's expected utility equals $r-\frac{1}{2}$ (i.e., her willingness-to-pay minus the expected time cost). Therefore, we define $\Delta_{m}=G_{m}-\left(r-\frac{1}{2}\right)$ as the optimal achievable surplus that can be secured by the optimal contract. Similarly, we define $\Delta_{l}^{m}=G_{l}^{m}-\left(r-\frac{1}{2}\right)$ as the available surplus achieved by the optimal FPFT contract. Using the results of Propositions 1 and 3, we evaluate the performance of the FPFT contract by calculating the performance ratio, $\frac{\Delta_{l}^{m}}{\Delta_{m}}$.

This measurement of performance ratio is both economically meaningful and free from the influence of (positive) affine transformations of the utility function. To illustrate, suppose that for some parameters the optimal contract and the FPFT contract, respectively, generate the expected utilities of 2 and 1 for the buyer, which implies a $50 \%$ decrease in expected utility. However, by adding 9 to the buyer's utility function, we could generate the expected utilities of 11 and 10, respectively. Now the decrease becomes $9 \%$, implying much poorer performance than $50 \%$. The term achievable surplus, however, is immune to this type of distortion.

Proposition 4 summarizes the performance of the FPFT contract in the multiplicative model.

Proposition 4 The performance ratio for the multiplicative model satisfies: a) $\frac{\Delta_{l}^{m}}{\Delta_{m}}=1$ if $k \geq \frac{1}{2}$; and $b$ ) $\frac{8}{9}<\frac{\Delta_{l}^{m}}{\Delta_{m}}<1$ if $0<k<\frac{1}{2}$.

Proposition 4 shows that the FPFT contract performs remarkably well over a wide range of parameters. In the worst case, the performance ratio is no less than $8 / 9$. To illustrate, we depict the performance ratio as a function of $k$ in Sub-figure 3(a). Sub-figure 3(b) shows that the optimal achievable surplus $\left(\Delta_{m}\right)$ decreases when the cost of effort increases. When the scalar $k$ approaches $1 / 2$ from the left, the performance ratio approaches $8 / 9$, and the optimal achievable surplus approaches 0 . Therefore, in terms of dollar values, the FPFT contract is nearly optimal. 


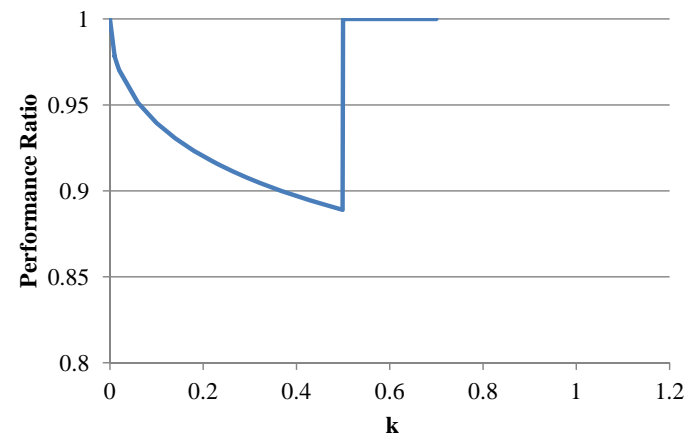

(a) Performance Ratio

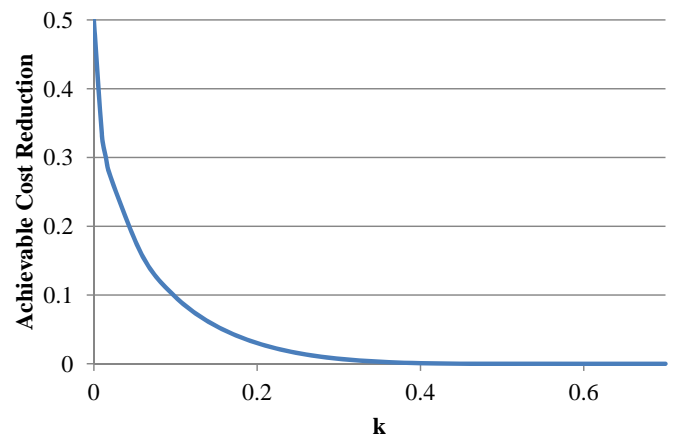

(b) Achievable Surplus

Figure 3: Performance of FPFT contract in the multiplicative model

By comparing the contract parameters in Propositions 1 and 3, we are able to further understand why the FPFT contract performs well. We concentrate on the case in which $0<k \leq \frac{1}{2}$.

Corollary 1 Assume $0<k \leq \frac{1}{2}$. Then we have: a) $2 k \leq t_{0}^{m}$; and b) $P_{0}^{m}=c\left(1-t_{0}^{m}\right) \leq P(0)=$ $\sqrt{2 k}+\sqrt{8 k^{3}}-4 k$ for $0<k \leq \frac{1}{2}$.

The proof of Corollary 1 is straightforward, and hence is omitted. Corollary 1 has two implications. First, the time threshold for the maximum payment in the optimal contract is slightly shorter than the maximum allowable time in the FPFT contract (where the equal sign holds if and only if $k=1 / 2$ ). In other words, the FPFT contract induces fewer supplier types to exert effort than does the optimal contract. Second, the fixed payment in the FPFT contract is smaller than the highest payment in the optimal contract. This means that the FPFT contract gives less information rent to the supplier types that do not exert time reduction efforts. When the maximum allowable time limit is set optimally, the rent reduction offsets the cost of delay. Therefore, the FPFT contract performs remarkably well in the multiplicative model.

\subsection{The Additive Model}

Now, we turn our attention to the additive model with $t(x, y)=x-y$. Similar to the multiplicative model, using Lemma 2, we derive the optimal FPFT contract in Proposition 5.

Proposition 5 The optimal FPFT contract for the additive model has the following properties. 
a) The buyer's optimal fixed time and fixed payment are determined by

$$
\left(t_{0}^{a}, P_{0}^{a}\right)= \begin{cases}(1,0), & h<\frac{1}{2}, \\ \left(0, \frac{1}{4 h}\right), & h \geq \frac{1}{2} .\end{cases}
$$

b) The buyer's optimal expected utility that can be achieved by implementing an FPFT contract is given by

$$
G_{l}^{a}= \begin{cases}r-\frac{1}{2}, & h<\frac{1}{2}, \\ r-\frac{1}{4 h}, & h \geq \frac{1}{2},\end{cases}
$$

which is continuous in $h$.

Proposition 5 shows a very simple FPFT contract for the additive model, which depends only on the effort cost parameterized by $h$. If the effort cost is high with $h<1 / 2$, then the buyer sets the maximum allow time to be 1 (i.e., $t_{0}^{a}=1$ ) and does not provide any incentive to the supplier (i.e., $P_{0}^{a}=0$ ). On the other hand, if the effort cost is low with $h \geq 1 / 2$, then the buyer motivates each type of the supplier to reduce the completion time to be 0 by paying a fixed payment of $1 /(4 h)$.

Similar to Sub-section 4.1, we use the achievable surplus as the benchmark. We define $\Delta_{a}=G_{a}-$ $\left(r-\frac{1}{2}\right)$ as the optimal achievable surplus secured by the optimal contract and $\Delta_{l}^{a}=G_{l}^{a}-\left(r-\frac{1}{2}\right)$ as the available surplus achieved by the optimal FPFT contract. Using the results of Propositions 2 and 5, we evaluate the performance of the FPFT contract by assessing the performance ratio, $\frac{\Delta_{l}^{a}}{\Delta_{a}}$.

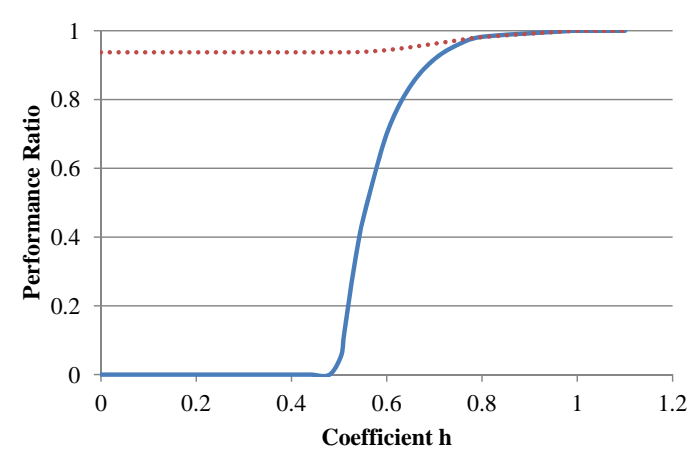

(a) Performance Ratio

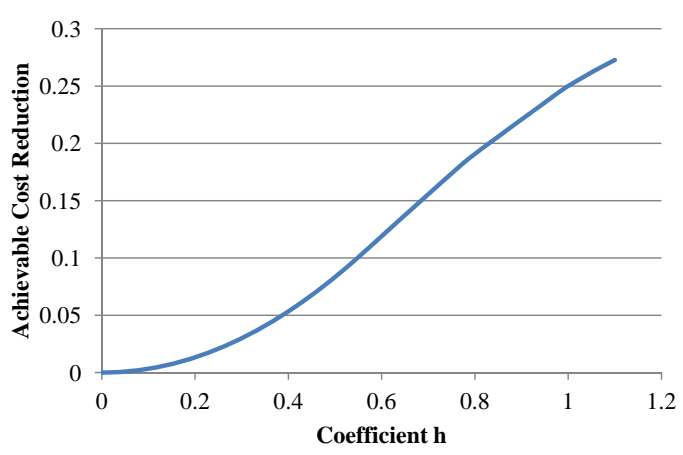

(b) Achievable Surplus

Figure 4: Performance of FPFT contract in the additive model

The solid line in Figure 4(a) depicts the performance ratio of the FPFT contract as a function of $h$. When $h \leq 1 / 2$, the cost of exerting effort is high and the performance ratio is zero. When $h$ approaches 1 , the performance ratio approaches 1 , which is consistent with part c) of Proposition 2 that an FPFT contract is 
optimal for $h \geq 1$. These observations suggest that the FPFT contract performs well only when the cost of exerting effort is small. The intuition of this result is the following: Recall that the optimal contract for the additive model induces the fast supplier to reduce time. In contrast, the FPFT contract induces the slow supplier to reduce time. When the cost of exerting effort is either moderate or high (e.g., $h \leq 0.7$ ), the performance of the FPFT contract worsens because the FPFT contract fails to motivate the right set of suppliers to reduce time.

In response to the underperformance of the FPFT contract in the additive model, we propose an enhanced linear contract as follows:

$$
P_{a}^{e}(t)= \begin{cases}\max \left(0, \frac{h-t}{2}\right), & 0<h<\frac{1}{2}, \\ \max \left(0,1-\frac{1}{4 h}-\frac{h}{2}-\frac{t}{2}\right), & \frac{1}{2} \leq h<1\end{cases}
$$

The above linear payment function resembles the optimal payment function derived in Proposition 2. For example, when $0<h<\frac{1}{2}$, the optimal payment function is $P_{a}(t)=\frac{h}{2}-\frac{t}{2}+\frac{t^{2}}{8 h}$, which resembles the linear function $\frac{h-t}{2}$ in equation (11). Clearly, the linear contract is able to induce the fast supplier to reduce time. Let $G_{a}^{e}$ denote the buyer's expected utility by implementing the aforementioned linear contract and $\Delta_{a}^{e}=G_{a}^{e}-\left(r-\frac{1}{2}\right)$ denote the available surplus. We summarize the performance of the enhanced linear contract in Proposition 6.

Proposition 6 The performance ratio of the enhanced linear contract in equation (11), $\frac{\Delta_{a}^{e}}{\Delta_{a}}$, is non-decreasing in $h$, and satisfies that $\frac{15}{16} \leq \frac{\Delta_{a}^{e}}{\Delta_{a}} \leq 1$ for $0<h<1$.

Proposition 6 reveals the remarkable performance of the enhanced linear contract. Using this contract, the buyer loses no more than $1 / 15$ of the available surplus secured by an optimal contract. The dotted line in Figure 4(a) depicts the performance ratio $\left(\frac{\Delta_{a}^{e}}{\Delta_{a}}\right)$ as a function of $h$. When $0<h<1 / 2, \frac{\Delta_{a}^{e}}{\Delta_{a}}=\frac{15}{16}$; when $1 / 2 \leq h<1, \frac{\Delta_{a}^{e}}{\Delta_{a}}$ monotonically increases from $15 / 16$ to 1 .

\section{CONCLUSION}

Given the importance of speed and time in current business world, more and more contracts involve clauses concerning completion time. In this study, we investigate the design of optimal time-based incentive when suppliers possess private information and take hidden action to reduce time. We demonstrate that different 
assumptions regarding time reduction could result in contrasting time-based incentive contracts. To avoid providing mismatched time incentive, it is imperative that the buyer gains a thorough understanding of the suppliers' time reduction effort. Our findings show that if the reduced time does not depend on the intrinsic time (suggesting that the time reduction may follow an additive model), the buyer should offer attractive bonuses to induce the intrinsically fast supplier to commit time reduction effort. If the reduced time depends on the intrinsic time (suggesting that the time reduction may follow a multiplicative model), the buyer should establish a firm deadline to motivate the intrinsically slow supplier to commit time-reduction effort.

Despite the fact that optimal contracts yield the maximum profit for the buyer, we admit that they are rarely used due to their complexity. Instead, fixed-price and fixed-time contracts are often observed in practice. A natural question is how well these simple contracts perform as opposed to the optimal contracts. To answer this question, we develop a performance ratio which is used to evaluate the performance of the FPFT contract. Our results show that the forgone achievable surplus is no more than one-ninth in the multiplicative model. However, the performance of an FPFT contract in the additive model deteriorates when the cost of exerting time reduction effort is medium or high, but is optimal when the cost of exerting time-reduction effort is sufficiently low. To tackle this inefficiency, we then propose an enhanced linear contract, and demonstrate that, using this contract, the buyer may lose no more than one-sixteenth of the achievable surplus in the additive model. These findings will help a time-sensitive buyer better design her time-based incentive, and highlight the importance of identifying time reduction forms.

This paper is the first attempt to study time-based incentive in operations management, and opens up several interesting extensions. First, we can relax our implicit assumption that the cost coefficients (e.g., $k$ and $h$ ) are known constants. An inquiry into this extension will likely involve the theory of multi-dimensional mechanism design, which remains less complete than that of one-dimensional mechanism design (Rochet and Stole, 2003). Second, our model considers only time-based incentive, thus, a model with time and other variables (e.g., order quantity or project quality) would be beneficial. For example, in a project management setting, a contractor may sacrifice quality in order to complete a project earlier, or it may take longer time to deliver quality outcomes to the buyer. Therefore, the question is how to design an incentive contract to induce both short time and good quality. We leave these extensions for future research. 


\section{APPENDIX: PROOFS OF LEMMAS AND PROPOSITIONS}

\section{Proof of Lemma 1}

Taking the derivative with respect to $x$ at both sides of equation (1), we find that

$$
\frac{\partial t(x, y(\hat{x} \mid x))}{\partial x}+\left.\frac{\partial t(x, y(\hat{x} \mid x))}{\partial y(\hat{x} \mid x)} \frac{d y(\hat{x} \mid x)}{d x}\right|_{\hat{x}=x}=0
$$

which implies that

$$
\left.\frac{d y(\hat{x} \mid x)}{d x}\right|_{\hat{x}=x}=-\frac{\frac{\partial t(x, y)}{\partial x}}{\frac{\partial t(x, y)}{\partial y}} .
$$

We write $U_{s}(x)=u_{s}(x \mid x)$ as the type- $x$ supplier's surplus in the truth-revealing equilibrium. The envelope theorem indicates that

$$
\frac{\partial U_{s}(x)}{\partial x}=\left.\frac{\partial u_{s}(\hat{x} \mid x)}{\partial x}\right|_{\hat{x}=x}=-\left.C^{\prime}(y(\hat{x} \mid x)) \cdot \frac{d y(\hat{x} \mid x)}{d x}\right|_{\hat{x}=x}=\frac{C^{\prime}(y(x)) \cdot \frac{\partial t(x, y)}{\partial x}}{\frac{\partial t(x, y)}{\partial y}},
$$

which is non-positive because of the regularity assumptions we impose on $C(\cdot)$ and $t(\cdot, \cdot)$. Thus, the individual reservation constraint implies that $U_{s}(1)=0$. Using this boundary condition, we obtain that

$$
U_{s}(x)=-\int_{x}^{1} U_{s}^{\prime}(\tilde{x}) d \tilde{x}=-\int_{x}^{1} C^{\prime}(y(\tilde{x})) \frac{\frac{\partial t(\tilde{x}, y(\tilde{x}))}{\partial t \tilde{x}}}{\frac{\partial t(\tilde{x}, y(\tilde{x}))}{\partial y(\tilde{x})}} d \tilde{x} .
$$

From the buyer's perspective, the supplier's ex-ante expected utility is given by

$$
\int_{0}^{1} U_{s}(x) d F(x)=\left.U_{s}(x) F(x)\right|_{0} ^{1}-\int_{0}^{1} F(x) U_{s}^{\prime}(x) d x .
$$

Because $F(0)=0$ and $U_{s}(1)=0$, we find that

$$
\begin{aligned}
\int_{0}^{1} U_{s}(x) d F(x) & =-\int_{0}^{1} F(x) U_{s}^{\prime}(x) d x=-\int_{0}^{1} \frac{C^{\prime}(y(x)) \cdot \frac{\partial t(x, y)}{\partial x}}{\frac{\partial t(x, y)}{\partial y}} F(x) d x \\
& =-\int_{0}^{1} \frac{C^{\prime}(y) \cdot \frac{\partial t(x, y)}{\partial x}}{\frac{\partial t(x, y)}{\partial y}} \frac{F(x)}{f(x)} d F(x) .
\end{aligned}
$$

Note that the buyer's ex-post utility is $r-t(x, y)-C(y)-U_{s}(x)$, where $U_{s}(x)$ is the net profit of the 
type- $x$ supplier. The buyer's ex-ante expected profit is given by

$$
\begin{aligned}
& \int_{0}^{1}\left[r-t(x, y)-C(y)-U_{s}(x)\right] d F(x) \\
= & \int_{0}^{1}\left[r-t(x, y)-C(y)+\frac{C^{\prime}(y) \cdot \frac{\partial t(x, y)}{\partial x} \frac{\partial t(x, y)}{\partial y}}{f(x)}\right] d F(x) \\
= & \int_{0}^{1} V(x, y) d F(x) .
\end{aligned}
$$

By maximizing the virtual surplus $V(x, y)$, we can maximize the buyer's expected profit. Furthermore, if $t\left(x, y^{*}(x)\right)$ is increasing in $x$, then the $y^{*}(x)$ function determined by equation (8) is the globally optimal solution to equation (3).

The payment to the type- $x$ supplier equals the sum of $U_{s}(x)$ and $C(y(x))$, which implies that

$$
P^{*}\left(t\left(x, y^{*}(x)\right)\right)=C\left(y^{*}(x)\right)+U_{s}(x)=C\left(y^{*}(x)\right)-\int_{x}^{1} C^{\prime}\left(y^{*}(\tilde{x})\right) \frac{\frac{\partial t(\tilde{x}, y(\tilde{x}))}{\partial \tilde{x}}}{\frac{\partial t(\tilde{x}, y(\tilde{x}))}{\partial y(\tilde{x})}} d \tilde{x} .
$$

\section{A Sufficient Condition}

It is more convenient to write the virtual surplus as a function of $t$ and $x$. Define a $g(t, x)$ function such that $t(x, g(t, x))=t$. Intuitively, $g(t, x)$ represents the effort level undertaken by a type- $x$ supplier to reduce the

completion time to be $t$. Therefore, we observe that $\frac{\partial g(t, x)}{\partial x}=-\frac{\frac{\partial t(x, y)}{\partial x}}{\frac{\partial t(x, y)}{\partial y}}$ and $\frac{\partial g(t, x)}{\partial t}=\frac{-1}{\frac{\partial t(x, y)}{\partial y}}$. Using these identities, the virtual surplus in equation (6) can be re-written as

$$
V(t, x)=r-t-C(g(t, x))-\frac{\partial C(g(t, x))}{\partial x} \frac{F(x)}{f(x)} .
$$

Note that the optimal realized time (which implicitly determines the optimal effort level) is determined by the following first-order condition:

$$
\frac{\partial V(t, x)}{\partial t}=-1-\frac{\partial C(g(t, x))}{\partial t}-\frac{F(x)}{f(x)} \cdot \frac{\partial}{\partial t}\left(\frac{\partial C(g(t, x))}{\partial x}\right)=0 .
$$

Using the implicit function theorem, we find that

$$
\frac{\partial t^{*}}{\partial x}=-\left.\frac{\frac{\partial^{2} V(t, x)}{\partial t \partial x}}{\frac{\partial^{2} V(t, x)}{\partial t^{2}}}\right|_{t=t^{*}}
$$


After performing some algebra, we obtain that

$$
-\frac{\frac{\partial^{2} V(t, x)}{\partial t \partial x}}{\frac{\partial^{2} V(t, x)}{\partial t^{2}}}=\frac{\frac{\partial^{2} C(g(t, x))}{\partial t \partial x} \cdot \frac{\partial t(x, y)}{\partial x} \cdot\left(1+\frac{\partial}{\partial x}\left(\frac{F(x)}{f(x)}\right)\right)+\frac{F(x)}{f(x)} \cdot \frac{\frac{\partial t(x, y)}{\partial x}}{\frac{\partial t(x, y)}{\partial y}} \cdot \frac{\partial}{\partial t}\left(\frac{\partial^{2} C(g(t, x))}{\partial x^{2}}\right)}{-\frac{\partial^{2} C(g(t, x))}{\partial t^{2}}+\frac{F(x)}{f(x)} \cdot \frac{1}{\frac{\partial t(x, y)}{\partial y}} \cdot \frac{\partial^{2}}{\partial t^{2}}\left(\frac{\partial C(g(t, x))}{\partial x}\right)} .
$$

Because $C(\cdot)$ is convex increasing, $\frac{\partial t(x, y)}{\partial x} \geq 0$, and $\frac{\partial t(x, y)}{\partial y} \leq 0$, we find that $\frac{\partial^{2} C(g(t, x))}{\partial t \partial x} \leq 0, \frac{\partial^{2} C(g(t, x))}{\partial t^{2}} \geq$ 0 , and $\frac{\partial^{2}}{\partial t^{2}}\left(\frac{\partial C(g(t, x))}{\partial x}\right) \geq 0$. We observe that the denominator in equation (14) is negative. A sufficient condition ensuring that the numerator in equation (14) is negative has two parts: 1) $\frac{\partial}{\partial x}\left(x+\frac{F(x)}{f(x)}\right)=$ $1+\frac{\partial}{\partial x}\left(\frac{F(x)}{f(x)}\right) \geq 0$, which means that $F(x)$ is log-concave; and 2) $\frac{\partial}{\partial t}\left(\frac{\partial^{2} c(g(t, x))}{\partial x^{2}}\right) \geq 0$. These two parts ensure that $\frac{\partial t^{*}}{\partial x} \geq 0$.

\section{Proof of Proposition 1}

a) By substituting $t(x, y)=x-x y, C(y)=\frac{k y^{2}}{1-y}, F(x)=x$, and $f(x)=1$ into equation (6) and after performing algebra, we find that the virtual surplus is

$$
V(x, y)=r-x+x y-\frac{2 k y}{(1-y)} .
$$

The first derivative of $V(x, y)$ with respect to $y$ is

$$
\frac{d}{d y}\left(r-x+x \cdot y-\frac{2 k y}{(1-y)}\right)=x-\frac{2 k}{(1-y)^{2}}
$$

The second derivative of $V(x, y)$ is

$$
\frac{d}{d y}\left(x-\frac{2 k}{(1-y)^{2}}\right)=\frac{-4 k}{(1-y)^{3}}<0
$$

for any $0 \leq y<1$. This implies that the virtual surplus is strictly concave with respect to $y$. Notice that if $x \leq 2 k$, then $\frac{d V(x, y)}{d y} \leq 0$ for all $y \in[0,1]$. Therefore, the optimal choice of effort is $y_{m}(x)=0$. If $x>2 k$, then the optimal choice of effort is $y_{m}(x)=1-\sqrt{\frac{2 k}{x}}$.

Notice that $t=x$ for $0 \leq x \leq 2 k$ and $t=x-x y_{m}(x)=\sqrt{2 k x}$ when $2 k \leq x \leq 1$. We observe that $t=x-x y_{m}(x)$ is continuous and increasing in $x$. Hence, the optimal effort function is globally optimal. In summary, the optimal effort induced by the buyer is $y_{m}(x)=\max \left(0,1-\sqrt{\frac{2 k}{x}}\right)$. 
b) The optimized virtual surplus is

$$
\begin{aligned}
V\left(x, y^{*}(x)\right) & =r-x+x\left(1-\sqrt{\frac{2 k}{x}}\right)-\frac{2 k\left(1-\sqrt{\frac{2 k}{x}}\right)}{1-\left(1-\sqrt{\frac{2 k}{x}}\right)} \\
& =r+2 k-\sqrt{8 k x}
\end{aligned}
$$

for $x \leq 2 k$; otherwise, $V\left(x, y^{*}(x)\right)=r-x$. We can compute the buyer's optimal utility as follows. When $k \geq \frac{1}{2}, y_{m}(x)=0$ for all $x \in[0,1]$, the buyer's utility is

$$
G_{m}=\int_{0}^{1}(r-x) d x=r-\frac{1}{2} .
$$

When $k \leq \frac{1}{2}$, the optimal effort is $y_{m}(x)=0$ for all $x \in[0,2 k]$ and $y_{m}(x)=1-\sqrt{\frac{2 k}{x}}$ for all $x \in[2 k, 1]$. Therefore, the buyer's utility is

$$
\begin{aligned}
G_{m} & =\int_{0}^{2 k}(r-x) d x+\int_{2 k}^{1}(r+2 k-\sqrt{8 k x}) d x \\
& =2 k r-2 k^{2}+2 k+r-2 k r-4 k^{2}-\frac{4}{3} \sqrt{2 k}+\frac{16}{3} k^{2} \\
& =r+2 k-\frac{2 k^{2}}{3}-\frac{4}{3} \sqrt{2 k} .
\end{aligned}
$$

c) Note that if $k \geq \frac{1}{2}$, then $y_{m}(x)=0$ for any $x \in[0,1]$. The buyer offers a fixed-price contract with $P(t)=0$ for any $t \in[0,1]$. When $k \leq \frac{1}{2}$, the supplier's surplus function is

$$
\begin{aligned}
U_{s}(x) & =\int_{x}^{1} C^{\prime}(y(\tilde{x}))\left(\frac{1-y_{m}(\tilde{x})}{\tilde{x}}\right) d \tilde{x} \\
& = \begin{cases}\sqrt{2 k}+\sqrt{8 k^{3}}-4 k & 0 \leq x \leq 2 k \\
\sqrt{2 k}+\sqrt{8 k^{3}}-\sqrt{2 k x}-\sqrt{\frac{8 k^{3}}{x}} & 2 k \leq x \leq 1 .\end{cases}
\end{aligned}
$$

The payment function satisfies

$$
\begin{aligned}
P\left(x-x y_{m}(x)\right) & =u(x)+C\left(y_{m}(x)\right) \\
& = \begin{cases}\sqrt{2 k}+\sqrt{8 k^{3}}-4 k & 0 \leq x \leq 2 k \\
\sqrt{2 k}+\sqrt{8 k^{3}}-\sqrt{\frac{k x}{2}}-\sqrt{\frac{2 k^{3}}{x}}-2 k & 2 k \leq x \leq 1 .\end{cases}
\end{aligned}
$$

Notice that $x=t$ for $0 \leq x \leq 2 k$. Equation (15) yields $P(t)=\sqrt{2 k}+\sqrt{8 k^{3}}-4 k$. When $2 k \leq x \leq 1$, $t=x-x y_{m}(x)=\sqrt{2 k x}$, equation (15) yields

$$
P(\sqrt{2 k x})=\sqrt{2 k}+\sqrt{8 k^{3}}-\sqrt{\frac{k x}{2}}-\sqrt{\frac{2 k^{3}}{x}}-2 k,
$$


which can be re-written as

$$
P(t)=\sqrt{2 k}+\sqrt{8 k^{3}}-\frac{t}{2}-\frac{2 k^{2}}{t}-2 k
$$

In summary, the optimal payment function is given by

$$
P_{m}(t)= \begin{cases}\sqrt{2 k}+\sqrt{8 k^{3}}-4 k & 0 \leq t \leq 2 k \\ \sqrt{2 k}+\sqrt{8 k^{3}}-\frac{t}{2}-\frac{2 k^{2}}{t}-2 k & 2 k \leq t \leq 1\end{cases}
$$

which is a continuous, non-increasing, and concave function.

\section{Proof of Proposition 2}

a) By substituting $t(x, y)=x-y, C(y)=\frac{y^{2}}{4 h}, F(x)=x$, and $f(x)=1$ into equation (6) and after performing algebra, we find that the virtual surplus is

$$
V(x, y)=r-x+y-\frac{y^{2}}{4 h}-\frac{y x}{2 h} .
$$

The second derivative of $V(x, y)$ with respect to $y$ equals $-\frac{1}{2 h}<0$. Solving the first order condition, we obtain the optimal effort function $y(x)$ as follows,

$$
y_{a}(x)=\left\{\begin{array}{lr}
x, & x \leq h, \\
2 h-x, & h \leq x \leq 2 h, \\
0, & x \geq 2 h .
\end{array}\right.
$$

Notice that

$$
x-y_{a}(x)=\left\{\begin{array}{lr}
0, & x \leq h, \\
2 x-2 h, & h \leq x \leq 2 h, \\
x, & x \geq 2 h,
\end{array}\right.
$$

which is non-decreasing in $x$. This observation means that the optimal effort function achieves complete ordering of the supplier's type. Hence, the $y_{a}(x)$ function is optimal.

b) Using equation (13), we find that when $h<\frac{1}{2}$,

$$
U_{s}(x)=\int_{x}^{1} C^{\prime}(y(\tilde{x})) d \tilde{x}=\left\{\begin{array}{lr}
0, & x \geq 2 h \\
h-x+\frac{x^{2}}{4 h}, & h \leq x \leq 2 h \\
\frac{h}{2}-\frac{x^{2}}{4 h}, & x \leq h .
\end{array}\right.
$$

It is readily seen that the buyer's expected utility is

$$
G_{a}=\int_{0}^{h}\left(r-\frac{x^{2}}{4 h}-\frac{x^{2}}{2 h}\right) d x+\int_{h}^{2 h}\left(r+h-2 x+\frac{x^{2}}{4 h}\right) d x+\int_{2 h}^{1}(r-x) d x=r+\frac{h^{2}}{3}-\frac{1}{2} .
$$


When $\frac{1}{2} \leq h<1$, we find that the supplier's surplus function is

$$
U_{s}(x)=\int_{x}^{1} C^{\prime}(y(\tilde{x})) d \tilde{x}=\left\{\begin{array}{lr}
\frac{1}{4 h} x^{2}-\frac{1}{4 h}-x+1, & h \leq x \leq 1, \\
1-\frac{1}{4 h}-\frac{1}{4 h} x^{2}-\frac{1}{2} h, & x \leq h,
\end{array}\right.
$$

and the buyer's expected utility is

$$
\begin{aligned}
G_{a} & =\int_{0}^{h}\left(r-\frac{x^{2}}{4 h}-\frac{x^{2}}{2 h}\right) d x+\int_{h}^{1}\left(r+h-2 x+\frac{x^{2}}{4 h}\right) d x \\
& =r+h+\frac{1}{12 h}-\frac{h^{2}}{3}-1 .
\end{aligned}
$$

Similarly, when $h \geq 1$, we find that the supplier's surplus function is

$$
U_{s}(x)=\int_{x}^{1} C^{\prime}(y(\tilde{x})) d \tilde{x}=\frac{1-x^{2}}{4 h},
$$

and the buyer's expected utility is

$$
G_{a}=\int_{0}^{1}\left(r-\frac{3 x^{2}}{4 h}\right) d x=r-\frac{1}{4 h}
$$

c) We obtain the payment function as follows.

$$
P(t)=U_{s}(x)+C\left(y_{a}(x)\right),
$$

where $U_{s}(x)$ function is derived in part b). When $0<h<\frac{1}{2}$, the optimal payment function satisfies

$$
P_{a}(t)= \begin{cases}0, & t \geq 2 h \\ \frac{h}{2}-\frac{t}{2}+\frac{t^{2}}{8 h} & t \leq 2 h\end{cases}
$$

When $\frac{1}{2} \leq h<1$, the optimal payment function satisfies

$$
P_{a}(t)= \begin{cases}0, & t>1-h \\ 1-\frac{1}{4 h}-\frac{h}{2}-\frac{t}{2}+\frac{t^{2}}{4 h} \quad t \leq 1-h .\end{cases}
$$

When $h \geq 1$, the optimal payment function satisfies $P_{a}(0)=\frac{1}{4 h}$ and $P_{a}(t)=0$ for any $t>0$.

\section{Proof of Lemma 2}

Under an arbitrary FPFT contract with time limit $t_{0}$ and payment $P_{0}$, the supplier whose intrinsic time is the longest (i.e., $x=1$ ) should receive zero surplus, which means that $P_{0}=C\left(y\left(t_{0}\right)\right)$, where $t\left(1, y\left(t_{0}\right)\right)=t_{0}$. Furthermore, the supplier whose intrinsic time is shorter than $t_{0}$ does not exert any time-reduction effort 
and all the suppliers whose intrinsic time is longer than $t_{0}$ will exert the right amount of effort such that the realized completion time exactly equals $t_{0}$. As such, the buyer's expected utility is

$$
G_{l}=\int_{0}^{t_{0}}(r-x) d F(x)+\int_{t_{0}}^{1}\left(r-t_{0}\right) d F(x)-C\left(y\left(t_{0}\right)\right) .
$$

The first order condition yields that $\frac{d C\left(y\left(t_{0}\right)\right)}{d t_{0}}=1-F\left(t_{0}\right)$, which determines the optimal fixed time limit. And then using the other two equations $P_{0}=C\left(y\left(t_{0}\right)\right)$ and $t\left(1, y\left(t_{0}\right)\right)=t_{0}$, we can identify the optimal fixed payment.

\section{Proof of Proposition 3}

a) By substituting $t(x, y)=x-x y, C(y)=\frac{k y^{2}}{1-y}, F(x)=x$, and $f(x)=1$ into equation (10) and after performing algebra, we find that the optimal fixed time limit is

$$
t_{0}^{m}= \begin{cases}\frac{k+\sqrt{4 k+k^{2}}}{2}, & k \leq \frac{1}{2}, \\ 1, & k \geq \frac{1}{2} .\end{cases}
$$

Consequently, the optimal fixed payment is given by $P_{0}^{m}=C\left(1-t_{0}^{m}\right)$.

b) Substituting $\left(t_{0}^{m}, P_{0}^{m}\right)$ into equation (16), we find that the buyer's expected utility that results from implementing an FPFT contract is

$$
\begin{aligned}
G_{l}^{m} & =-C\left(1-t_{0}^{m}\right)+\int_{0}^{t_{0}^{m}}(r-x) d F(x)+\int_{t_{0}^{m}}^{1}\left(r-t_{0}^{m}-C\left(1-t_{0}^{m}\right)\right) d F(x) \\
& =r+2 k-t_{0}-k t_{0}+\frac{1}{2} t_{0}^{2}-\frac{k}{t_{0}} \\
& = \begin{cases}r+\frac{5}{2} k-\frac{k^{2}}{4}-\frac{k+4}{4} \sqrt{4 k+k^{2}} & k \leq \frac{1}{2} \\
r-\frac{1}{2} & k \geq \frac{1}{2} .\end{cases}
\end{aligned}
$$

\section{Proof of Proposition 4}

Using the results of Propositions 1 and 3, we find that when $k=0$ or $k \geq \frac{1}{2}$, it satisfies that $\frac{\Delta_{l}^{m}}{\Delta_{m}}=1$ because $G_{l}^{m}=G_{m}=r-\frac{1}{2}$. We concentrate on the case in which $0<k<\frac{1}{2}$. The performance ratio $\frac{\Delta_{l}^{m}}{\Delta_{m}}$ can be written as

$$
\frac{\Delta_{l}^{m}}{\Delta_{m}}=\frac{\frac{1}{2}+\frac{5 k}{2}-\frac{1}{4} k^{2}-\frac{k+4}{4} \sqrt{4 k+k^{2}}}{\frac{1}{2}+2 k-\frac{2}{3} k^{2}-\frac{4}{3} \sqrt{2 k}} .
$$

We observe that $\lim _{k \rightarrow 1} \frac{\Delta_{l}^{m}}{\Delta_{m}}=1$. Using L'Hospital's rule three times, we find that

$$
\lim _{k \rightarrow \frac{1}{2}} \frac{\Delta_{l}^{m}}{\Delta_{m}}=\lim _{k \rightarrow \frac{1}{2}} \frac{-\Theta}{-\frac{\sqrt{2}}{(2 k)^{\frac{5}{2}}}}=\frac{-\frac{32}{9}}{-4}=\frac{8}{9}
$$


where $\Theta=\frac{d^{3}}{d k^{3}}\left(\frac{k+4}{4} \sqrt{4 k+k^{2}}\right)$, which is very lengthy and therefore omitted. Furthermore, it can be verified that $\frac{\Delta_{l}^{m}}{\Delta_{m}}$ is non-increasing with respect to $k$ when $k \in\left[0, \frac{1}{2}\right)$. Figure 3 precisely plots $\frac{\Delta_{l}^{m}}{\Delta_{m}}$ as a function of $k$ and confirms this monotonic property. Therefore, we conclude that when $0<k<\frac{1}{2}$, the performance ratio $\frac{\Delta_{l}^{m}}{\Delta_{m}}$ is between $\frac{8}{9}$ and 1 .

\section{Proof of Proposition 5}

a) By substituting $t(x, y)=x-y, C(y)=\frac{y^{2}}{4 h}, F(x)=x$, and $f(x)=1$ into equation (16) and after some algebra, we find that

$$
G_{l}^{a}=t_{0}^{2}\left(\frac{1}{2}-\frac{1}{4 h}\right)-2 t_{0}\left(\frac{1}{2}-\frac{1}{4 h}\right)+r-\frac{1}{4 h} .
$$

When $h<\frac{1}{2}, G_{l}^{a}$ is concave in $t_{0}$; the first-order condition yields $t_{0}^{a}=1$. When $h \geq \frac{1}{2}, G_{l}^{a}$ is convex in $t_{0}$; the optimizer is $t_{0}^{a}=0$. Using the condition $P_{0}^{a}=C\left(1-t_{0}^{a}\right)$, we find the optimal fixed payment.

b) Substituting the optimal fixed time and fixed payment into equation (16), we find that the buyer's expected utility that results from implementing an FPFT contract is given by

$$
G_{l}^{a}= \begin{cases}r-\frac{1}{2}, & h<\frac{1}{2} \\ r-\frac{1}{4 h}, & h \geq \frac{1}{2}\end{cases}
$$

which is continuous in $h$.

\section{Proof of Proposition 6}

First, consider that $0<h<\frac{1}{2}$. The linear payment function is $\left(\frac{h-t}{2}\right)^{+}$, where $(\cdot)^{+}=\max (0, \cdot)$. A type- $x$ supplier's utility is

$$
U_{s}(x)=\max _{y \leq x}\left\{\frac{(h-x+y)^{+}}{2}-\frac{y^{2}}{4 h}\right\} .
$$

After solving the first order condition, we find that the best response of a type- $x$ supplier is to choose an effort level of $y(x)=x$ if $x \leq h, y(x)=h$ if $h \leq x \leq \frac{3 h}{2}$, and $y(x)=0$ if $x \geq \frac{3 h}{2}$. Recall that $h<\frac{1}{2}$, the upper limit of the integral is $\frac{3 h}{2}<\frac{3}{4}$. Therefore, the buyer's utility by implementing the linear contract is

$$
G_{a}^{e}=\int_{0}^{h}\left(r-\frac{h}{2}\right) d x+\int_{h}^{\frac{3 h}{2}}\left(r-x+h-\frac{h}{2}+\frac{x-h}{2}\right) d x+\int_{\frac{3 h}{2}}^{1}(r-x) d x=r-\frac{1}{2}+\frac{5}{16} h^{2} .
$$

Using part b) of Proposition 2, we observe that the performance ratio is

$$
\frac{\Delta_{a}^{e}}{\Delta_{a}}=\frac{r-\frac{1}{2}+\frac{5}{16} h^{2}-\left(r-\frac{1}{2}\right)}{r-\frac{1}{2}+\frac{1}{3} h^{2}-\left(r-\frac{1}{2}\right)}=\frac{15}{16} .
$$


Next, we consider that $\frac{1}{2} \leq h<1$. The linear payment function is $\left(1-\frac{1}{4 h}-\frac{h}{2}-\frac{t}{2}\right)^{+}$. After solving the first order condition, we find that the best response of a type- $x$ supplier is to choose an effort level of $y(x)=x$ if $x \leq h, y(x)=h$ if $h \leq x \leq 2-\frac{1}{2 h}-\frac{h}{2}$, and $y(x)=0$ if $x \geq 2-\frac{1}{2 h}-\frac{h}{2}$. Recall that $\frac{1}{2} \leq h<1$, the upper limit of the integral is $2-\frac{1}{2 h}-\frac{h}{2}<1$. Therefore, the buyer's utility by implementing the linear contract is

$$
\begin{aligned}
G_{a}^{e} & =\int_{0}^{h}\left(r-1+\frac{1}{4 h}+\frac{h}{2}\right) d x+\int_{h}^{2-\frac{1}{2 h}-\frac{h}{2}}\left(r-x+h-1+\frac{1}{4 h}+\frac{h}{2}+\frac{x-h}{2}\right) d x \\
& +\int_{2-\frac{1}{2 h}-\frac{h}{2}}^{1}(r-x) d x=r-2+2 h+\frac{1}{2 h}-\frac{1}{16 h^{2}}-\frac{11}{16} h^{2} .
\end{aligned}
$$

Using part b) of Proposition 2, we observe that the performance ratio is

$$
\frac{\Delta_{a}^{e}}{\Delta_{a}}=\frac{r-2+2 h+\frac{1}{2 h}-\frac{1}{16 h^{2}}-\frac{11}{16} h^{2}-\left(r-\frac{1}{2}\right)}{r+h+\frac{1}{12 h}-\frac{h^{2}}{3}-1-\left(r-\frac{1}{2}\right)}=\frac{2 h+\frac{1}{2 h}-\frac{1}{16 h^{2}}-\frac{11}{16} h^{2}-\frac{3}{2}}{h+\frac{1}{12 h}-\frac{1}{3} h^{2}-\frac{1}{2}} .
$$

One can verify that the above equation is increasing in $h$. When $h \rightarrow \frac{1}{2}, \frac{\Delta_{a}^{e}}{\Delta_{a}} \rightarrow \frac{15}{16}$; when $h \rightarrow 1, \frac{\Delta_{a}^{e}}{\Delta_{a}} \rightarrow 1$. In summary, the performance ratio $\frac{\Delta_{a}^{e}}{\Delta_{a}}$ is weakly increasing in $h$ from $\frac{15}{16}$ to 1 .

\section{REFERENCES}

Barron, D. P. and Myerson, R. B. 1982. "Regulating a monopolist with unknown costs." Econometrica, 50(4), 911-930.

Baxter, J. B. 1994. "Responding to the Northbridge earthquake." PM Network, November Issue, 12-22.

Bolton, P. and Dewatripont, M. 2005. "Contract Theory", The MIT Press, MA, USA.

Cachon, G. P., \& Zhang, F. 2006. "Procuring fast delivery: Sole sourcing with information asymmetry." Management Science, 52(6), 881-896.

Che, Y.-K. 1993. "Design competition through multidimensional auctions.” RAND Journal of Economics, 24(4), 668-680.

Chen-Ritzo, C.-H., Harrison, T., Kwasnica, A., \& Thomas, D. 2005. "Better, faster, cheaper: An experimental analysis of a multiattribute reverse auction mechanism with restricted information feedback." Management Science, 51(12):1753-1762. 
Dai, T., Cho, S.-H., and Zhang, F. 2012. "Contracting for on-time delivery in the U.S. influenza vaccine supply chain.” Available at SSRN: http://ssrn.com/abstract=2178157.

Grout, J. R., and Christy, D. P. 1993. "An inventory model of incentives for on-time delivery in just-in-time purchasing contracts." Naval Research Logistics, 40(6), 863-877.

Grout, J. R. 1998. "Influencing a supplier using delivery windows: Its effect on the variance of flow time and on-time delivery." Decision Sciences, 29(3): 747-764.

Hendricks, K. B., and Singhal, V. R. 1997. "Delays in new product introductions and the market value of the firm: The consequences of being late to the market." Management Science, 43(4), 422-436.

Hendricks, K. B. and Singhal, V. R. 2008. "The effect of product introduction delays on operating performance." Management Science, 54(5), 878-892.

Holmstrom, B., and Milgrom, P. 1987. "Aggregation and linearity in the provision of intertemporal incentives." Econometrica, 55(2), 302-328.

Jayaram, J. and Malhotra, M. K. 2010. "The differential and contingent impact of concurrency on new product development project performance: A holistic examination.” Decision Sciences, 41(1): 147-196.

Konchar, M., and Sanvido, V. 1998. "Comparison of U.S. project delivery systems.” Journal of Construction Engineering Management, 124(6), 435-444.

Laffont, J.-J., and Tirole, J. 1986. "Using cost observation to regulate firms.” Journal of Political Economy, 94(3), 614-641.

Larson, E. W. and Gray, C. F. 2011. "Project management: The managerial process." 5th Edition, McGraw Hill/Irwin, New York.

Lerner, M. 2002. “Outsourcing in bio-technology pickups speed.” Chemical Market Reporter, 251(14), p. 17.

Lewis, G. and Bajari, P. 2011. "Procurement contracting with time incentives: Theory and evidence." The Quarterly Journal of Economics, 126(3): 1173-1211.

Rogerson, W. P. 2003. "Simple menus of contracts in cost-based procurement and regulation." American Economic Review, 93(3): 919-926. 


\section{ONELINE SUPPLEMENT}

In this Online Supplement, we discuss several extensions for the paper "Time-Based Procurement".

\section{Time-Based Auction}

We extend the analysis to the case with $N \geq 1$ suppliers competing for the buyer's business. Following the standard auction literature (Krishna, 2009), we assume that these $N$ suppliers are homogeneous in the sense that they have the same $t(x, y)$ and $C(y)$ functions. However, their private information regarding the intrinsic completion time is identically and independently distributed (i.i.d.) with $\operatorname{CDF} F(x)$ and $\operatorname{PDF} f(x)$. The buyer's challenge is to determine i) a selection rule such that the fastest supplier always wins; and ii) a payment function $P(\cdot)$ such that she makes a payment of $P(t)$ to the winner under the condition that the winner delivers the item at time $t$. If a type- $x$ supplier reports his type as $\hat{x}$, then he wins the auction when all of the other suppliers report their types greater than $\hat{x}$, which occurs with probability $(1-F(\hat{x}))^{N-1}$. As such, we find that a type- $x$ supplier who reports his type to be $\hat{x}$ attains an expected surplus of

$$
U(\hat{x} \mid x)=(1-F(\hat{x}))^{N-1}[P(t(\hat{x}, y(\hat{x})))-C(y(\hat{x} \mid x))]
$$

In the truth-revealing equilibrium, the individual reservation constraint requires $U(x \mid x) \geq 0$ for all $x \in$ $[0,1]$, and the incentive compatibility constraint requires $U(x \mid x) \geq U(\hat{x} \mid x)$ for all $\hat{x}, x \in[0,1]$.

Let $x_{(1)}$ be the shortest intrinsic time of these $N$ suppliers. The CDF and PDF of $x_{(1)}$ are $F_{(1)}(x)=$ $1-(1-F(x))^{N}$ and $f_{(1)}(x)=N f(x)(1-F(x))^{N-1}$, respectively. Lemma 3 derives an upper bound on the buyer's expected utility with the presence of incomplete information and hidden information.

Lemma 3 When there are $N \geq 1$ suppliers available, the highest expected utility that the buyer can attain among all feasible procurement strategies is

$$
G_{U}=\int_{0}^{1} V\left(x, y^{*}(x)\right) d F_{(1)}(x),
$$

where $V(x, y)$ is the virtual surplus function given by equation (6) and $y^{*}(x)$ is the optimal effort function determined by equation ( 8 ). 
Proof. Taking the first order derivative of $U(\hat{x} \mid x)$ with respect to $x$ and evaluating it at $\hat{x}=x$, we obtain

$$
U^{\prime}(x)=\left.\frac{\partial U(\hat{x} \mid x)}{\partial x}\right|_{\hat{x}=x}=(1-F(x))^{N-1} C^{\prime}(y(x)) \frac{\frac{\partial t(x, y)}{\partial x}}{\frac{\partial t(x, y)}{\partial y}} .
$$

The individual rationality constraint implies that $U(1)=0$. Thus, we have $U(x)=-\int_{x}^{1} U^{\prime}(\tilde{x}) d \tilde{x}$.

The ex-ante utility of a randomly chosen supplier is

$$
\int_{0}^{1} U(x) d F(x)=\left.U(x) F(x)\right|_{0} ^{1}-\int_{0}^{1} F(x) U^{\prime}(x) d x=-\int_{0}^{1} F(x) U^{\prime}(x) d x,
$$

where the last equality follows $U(1)=0$ and $F(0)=0$. Therefore, we have

$$
\begin{aligned}
\int_{0}^{1} U(x) d F(x) & =-\int_{0}^{1} C^{\prime}(y(x)) \frac{\frac{\partial t(x, y)}{\partial x}}{\frac{\partial t(x, y)}{\partial y}}(1-F(x))^{N-1} \frac{F(x)}{f(x)} d F(x) \\
& =-\frac{1}{N} \int_{0}^{1} C^{\prime}(y(x)) \frac{\frac{\partial t(x, y)}{\partial x}}{\frac{\partial t(x, y)}{\partial y}} \frac{F(x)}{f(x)} d F_{(1)}(x) .
\end{aligned}
$$

The buyer's ex-ante utility equals the expected supply chain surplus minus the ex-ante information rent paid to the suppliers. We find that

$$
\begin{aligned}
G & =\int_{0}^{1} r-t(x, y)-C(y) d F_{(1)}(x)-N \int_{0}^{1} U(x) d F(x) \\
& =\int_{0}^{1} r-t(x, y)-C(y)+\frac{C^{\prime}(y) \cdot \frac{\partial t(x, y)}{\partial x}}{\frac{\partial t(x, y)}{\partial y}} \frac{F(x)}{f(x)} d F_{(1)}(x) \\
& =\int_{0}^{1} V(x, y) d F_{(1)}(x) \leq \int_{0}^{1} V\left(x, y^{*}(x)\right) d F_{(1)}(x)=G_{U} .
\end{aligned}
$$

An important observation of Lemma 3 is that competition does not induce more time reduction effort from the selected supplier because the optimal effort function $y^{*}(x)$ remains unchanged.

The remaining question is how to achieve the optimal procurement outcome described in Lemma 3. Laffont and Tirole (1987) provide two alternatives. The procedures of the first alternative are the following. Each supplier is first asked to report an effortless completion time (which equals the intrinsic time if truthrevealing is the dominating strategy). The supplier who reports the fastest effortless completion time is selected as the winner. Next, the buyer asks the winner (who reports $x$ ) to commit some extra time reduction effort according to the $y^{*}(x)$ function determined by equation (8). The winner receives a final payment that 
equals

$$
C\left(y^{*}(x)\right)-\int_{x}^{x_{(2)}} C^{\prime}\left(y^{*}(\tilde{x})\right) \frac{\frac{\partial t\left(\tilde{x}, y^{*}(\tilde{x})\right)}{\partial \tilde{x}}}{\frac{\partial t\left(\tilde{x}, y^{*}(\tilde{x})\right)}{\partial y^{*}(\tilde{x})}} d \tilde{x},
$$

where $x_{(2)}$ is the second fastest effortless completion time. Laffont and Tirole prove that truth-revealing is the dominating strategy for all the suppliers and the buyer's expected utility equals $G_{U}$. The first alternative of Laffont and Tirole has two disadvantages. First, the payment received by the selected supplier is random (because the payment depends on the second fastest time reported in the auction). Second, the supplier may concern about a potential renegade of the buyer. For instance, after the supplier truthfully reports his effortless completion time, the buyer forces the winner to take the chain-optimal time reduction effort $\bar{y}(x)$ instead of $y^{*}(x)$ but only agrees to cover the disutility incurred (i.e., $P(x)=C(\bar{y}(x))$ ).

The second alternative proposed by Laffont and Tirole uses the following procedures. The buyer announces a payment function $P^{*}(t)$, which is determined by equation (9). Each supplier then bids for the right to execute this contract. The supplier who bids the highest upfront fee wins the auction and pays the second highest upfront fee to the buyer (i.e., a Vickery type auction). After that, the winner fulfills the contract and receives the final payment according to the pre-announced $P^{*}(t)$ function. Because the winner can freely choose the delivery time based on the pre-announced payment function, the concern of a potential renegade of the buyer diminishes. But a disadvantage of the second alternative is that the selected supplier needs to pay an up front fee, which could be unattractive to a cash-constrained supplier.

The second alternative of Laffont and Tirole is similar to the supply contract auction studied by Chen (2007). To illustrate, consider that time reduction follows an additive model and the cost function is $C(y)=$ $\frac{y^{2}}{4 h}$ with $h \geq 1$. Part c) of Proposition 2 indicates that the optimal contract is a fixed price and fixed time contract with a fixed price of $\frac{1}{4 h}$ and a fixed time of 0 . A type- $x$ supplier, if he wins the auction, attains a surplus of $\frac{1-x^{2}}{4 h}$. In a Vickery auction, a type- $x$ supplier submits a bid of $\frac{1-x^{2}}{4 h}$. The winner pays the second highest upfront fee to the supplier. As such, the buyer's expected utility is

$$
r-\frac{1}{4 h}+\int_{0}^{1} \frac{1-x^{2}}{4 h} d F_{(2)}(x)=r-\frac{3}{h\left(4+6 N+2 N^{2}\right)} .
$$

On the other hand, using equation (17), we find that

$$
G_{U}=\int_{0}^{1} r-\frac{3 x^{2}}{4 h} d F_{(1)}(x)=r-\frac{3}{h\left(4+6 N+2 N^{2}\right)} .
$$

Clearly, the supply contract auction achieves the optimal procurement outcome. 


\section{An Extension with Concave Utility}

We emphasize that the virtual surplus $V(x, y)$ in equation (6) does not rely on the linearity of the buyer's utility function. Suppose that the buyer's utility is $r-t-b t^{2}$, where $b \geq 0$ is a constant and $t$ is the realized time. This utility function implies that a longer delay is more costly to the buyer. We find that the virtual surplus equals

$$
V(x, y)=r-t(x, y)-b(t(x, y))^{2}-C(y)+\frac{C^{\prime}(y) \cdot \frac{\partial t(x, y)}{\partial x}}{\frac{\partial t(x, y)}{\partial y}} \frac{F(x)}{f(x)}
$$

In the additive model with $C(y)=\frac{y^{2}}{4 h}$, the virtual surplus is

$$
V(x, y)=r-(x-y)-b(x-y)^{2}-\frac{y^{2}}{4 h}-\frac{x y}{2 h} .
$$

After optimizing the above virtual surplus function, we find that the optimal effort level is

$$
y_{a}(x)= \begin{cases}x, & x \leq h \\ \frac{2 h-(1-4 b h) x}{1+4 b h}, & h \leq x \leq \frac{2 h}{1-4 b h} \\ 0, & x \geq \frac{2 h}{1-4 b h}\end{cases}
$$

Note that $\frac{2 h}{1-4 b h} \geq 2 h$, where the equal sign holds if and only if $b=0$. Equation (18) indicates that the optimal effort function exhibits the same pattern as it does in the linear case where $b=0$.

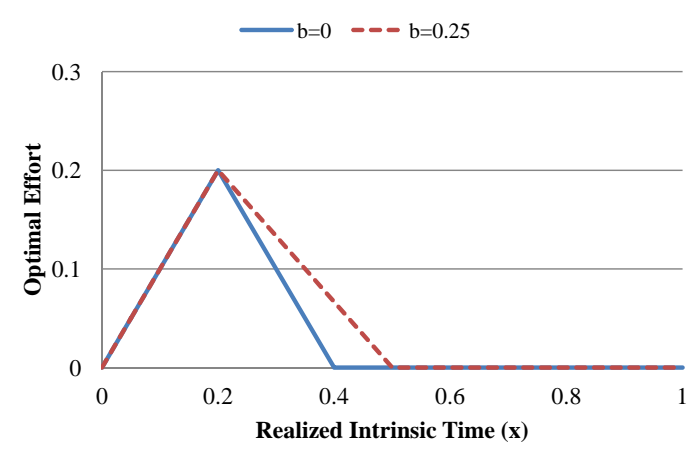

(a) Optimal Effort Induced By Buyer

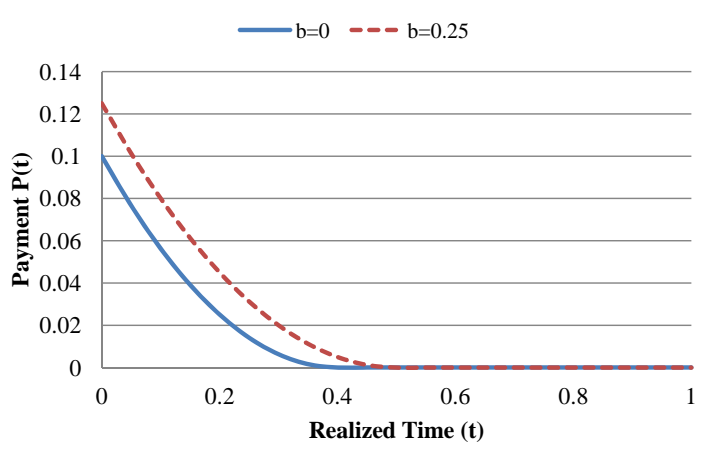

(b) Optimal Payment Function

Figure 5: Properties of optimal contract for the additive model

Using $h=0.2$, we depict the optimal effort and payment function for the additive model in Figure 5. The dotted curve indicates that when a longer delay is more costly, the buyer attempts to induce the intrinsically fast supplier to exert more time-reduction efforts as compared with in the linear case. 
Similarly, in the multiplicative model with $C(y)=\frac{k y^{2}}{1-y}$, we find that the virtual surplus is

$$
V(x, y)=r-(x-x y)-b(x-x y)^{2}-\frac{2 k y}{(1-y)} .
$$

Optimizing the above virtual surplus, however, would require to solve a cubic equation.

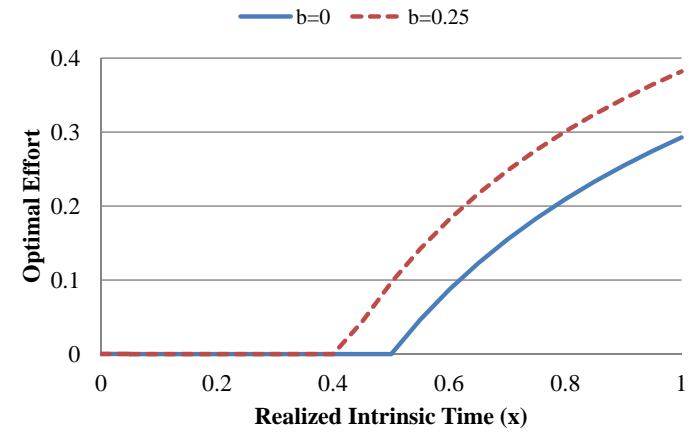

(a) Optimal Effort Induced By Buyer

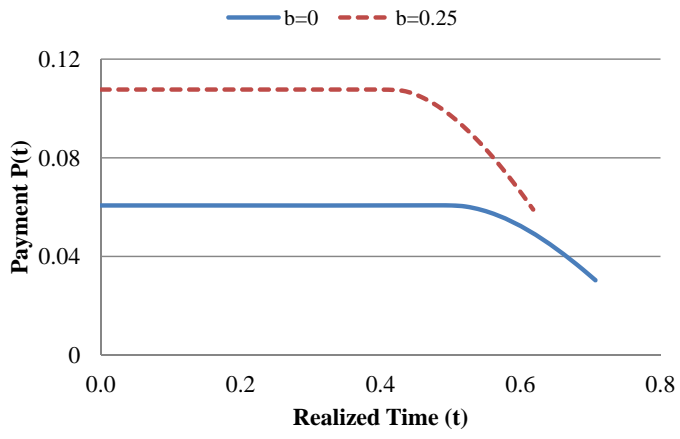

(b) Optimal Payment Function

Figure 6: Properties of optimal contract for the multiplicative model

Using $k=0.25$, we depict the optimal effort and payment function for the additive model in Figure 6 . Again, we observe that when a longer delay is more costly (see the dotted curve associated with $b=0.25$ ), the buyer attempts to induce the intrinsically slow supplier to exert more time reduction efforts than in the linear case.

\section{An Extension with Unknown Cost of Effort}

We now discuss an extension that presumes that the intrinsic time is known (and normalized to be 1) but the cost of exerting effort is private. Because the intrinsic time is fixed, the additive and multiplicative time reduction models are identical (i.e., $t(1, y)=1-y$ in both models). The issue of additive or multiplicative time-reduction model diminishes in this extension. We now illustrate how to derive the virtual surplus. Assume that the cost of exerting effort is $C(y)=\frac{y^{2}}{4 h}$, where $h$ is randomly distributed over the interval $\left[h_{1}, h_{2}\right]$ with $\operatorname{CDF} \Phi(\cdot)$ and $\operatorname{PDF} \phi(\cdot)$. If a type- $h$ supplier pretends to be type- $\hat{h}$, he must commit the same level of effort such that $t(1, y(\hat{h} \mid h))=t(1, y(\hat{h}))$, which implies that $y(\hat{h} \mid h)=y(\hat{h})$. By pretending to be type- $\hat{h}$, the supplier attains a surplus of $u_{s}(\hat{h} \mid h)=P(t(\hat{h}))-C(y(\hat{h} \mid h))$. We write $U_{s}(h)=u_{s}(\hat{h} \mid h)$ as the 
type- $h$ supplier's surplus in the truth-revealing equilibrium. The envelope theorem indicates that

$$
\frac{\partial U_{s}(h)}{\partial h}=\left.\frac{\partial u_{s}(\hat{h} \mid h)}{\partial h}\right|_{\hat{h}=h}=-C^{\prime}(y(h)) .
$$

Using the same procedure as in the proof of Lemma 1, we can derive the virtual surplus as

$$
V(h, y)=r-(1-y)-\frac{y^{2}}{4 h}-\frac{y}{2 h} \frac{\Phi(h)}{\phi(h)} .
$$

We observe that the virtual surplus function is concave in $y$ for any given $h$. Using the point-wise optimization procedure, we can find the optimal effort and payment function.

\section{REFERENCES}

Chen, F. 2007. “Auctioning supply contract.” Management Science, 53(10),1562-1576.

Krishna V. 2009. "Auction theory”, 2nd Edition, Academic Press, MA, USA.

Laffont, J.-J., and Tirole, J. 1987. "Auctioning incentive contracts.” Journal of Political Economy, 95(5), 921-937. 


\section{University Library}

\section{- M M I E E R VA A gateway to Melbourne's research publications}

Minerva Access is the Institutional Repository of The University of Melbourne

Author/s:

Li, Z;Shao, L

Title:

Time-Based Procurement

Date:

2015

Citation:

Li, Z. \& Shao, L. (2015). Time-Based Procurement. International Journal of Production Economics, 169 (November), pp.350-361. https://doi.org/10.1016/j.ijpe.2015.08.009.

Persistent Link:

http://hdl.handle.net/11343/116301 